\title{
RANK THREE AFFINE PLANES
}

\author{
MICHAEL J. KALLAHER ${ }^{1}$
}

\begin{abstract}
ABSTRACr. A permutation group has rank 3 if it is transitive and the stabilizer of a point has exactly three orbits. A rank 3 collineation group of an affine plane is one which is a rank 3 permutation group on the points. Several people (see [4], [7], [8], [12]) have characterized different kinds of affine planes using rank 3 collineation groups. In this article we prove the following: Let $\mathscr{A}$ be a finite affine plane of nonsquare order having a rank 3 collineation group which acts regularly on one of its orbits on the line at infinity. $\mathscr{A}$ must be either (i) a Desarguesian plane, (ii) a semifield plane, or (iii) a generalized André plane.
\end{abstract}

1. Introduction. A permutation group is of rank 3 if it is transitive and the stabilizer of a point $P$ has three orbits (including $\{P\}$ ). A rank 3 collineation group on an affine plane is a collineation group of the plane that is of rank 3 when considered as a permutation group on the points of the plane. The following holds:

THEOREM 1. If $\mathscr{A}$ is a finite affine plane of order $n$ admitting a rank 3 collineation group $G$, then $\mathscr{A}$ is a translation plane, $n=p^{r}$ for some prime $p$ and integer $r \geqq 1$, and $G$ contains the group of translations of $\mathscr{A}$.

This was first stated by Higman [4] but a proof was given only under the condition that $G$ was transitive on the line at infinity. However $G$ may also have two orbits on the line at infinity; the proof for this case was given with slight restrictions by Kallaher [6] and in full generality by Liebler [9].

Examples of rank 3 affine planes, besides Desarguesian planes, include the Lüneburg planes [10], the nearfield planes [6], certain semifield planes [7], certain generalized André planes [8], and the Hall planes [12]. Except for the Lüneburg planes, in each of the explicit examples, the rank 3 collineation group given has the property that it induces a regular (sharply transitive) permutation group on one of its orbits on the line at infinity $l_{\infty}$ of the affine plane.

Higman [4] proved that if the rank 3 collineation group $G$ acts regularly on the line at infinity then the plane is Desarguesian. Prohaska [12] gives

Received by the editors September 13, 1971.

AMS 1970 subject classifications. Primary 05B25, 50D35; Secondary 20B25.

Key words and phrases. Affine plane, collineation group, rank 3.

${ }^{1}$ Supported in part by NSF Grant GP-27101. 
the following characterization of the Hall planes: Let $\mathscr{A}$ be an affine plane of square order $n=m^{2} . \mathscr{A}$ is a Hall plane if and only if $\mathscr{A}$ admits a rank 3 collineation group $G$ having an orbit of length $m+1$ on $l_{\infty}$ upon which $G$ induces a regular permutation group.

With these two results in mind, in this article we investigate planes with rank 3 collineation groups having an orbit on $l_{\infty}$ upon which they induce a regular permutation group. Our main result is:

MAIN THEOREM. Let $\mathscr{A}$ be a finite affine plane of order $n$ with a rank 3 collineation group $G$. If $n$ is not a square and $G$ induces a regular permutation group on one of its orbits on the line at infinity, then $\mathscr{A}$ is one of the following three types of planes: (i) Desarguesian plane; (ii) semifield plane; (iii) generalized André plane. (See Corollary 3.1.)

Comparing this result with Prohaska's, it is reasonable to conjecture that if $n$ is a square, besides types (i)-(iii), the only other type possible is (iv) Hall plane.

We assume the reader is familiar with the basic terminology, notation, and theory of affine and projective planes as is contained in Dembowski [3]. We will use $l_{\infty}$ to denote the line at infinity of an affine plane. We also presume the reader knows the fundamentals of permutation groups as contained in Passman [11]. In particular, by a group $G$ acting regularly on an orbit $\Gamma$ we mean that $P \sigma=P, P \in \Gamma$ and $\sigma \in G$ implies $\sigma$ fixes every point of $\Gamma$.

2. Preliminaries. Let $\mathscr{A}$ be an affine plane of order $n$ with a rank 3 collineation group $G$. By Theorem $1, \mathscr{A}$ is a translation plane and hence $n=p^{r}$ for some prime $p$ and some integer $r \geqq 1$. Furthermore $G$ contains the group $T$ of translations of $\mathscr{A}$ and thus $G=T G_{O}$ where $O$ is a point of $\mathscr{A}$. This implies $G$ and $G_{O}$ induce the same permutation group on $l_{\infty}$, the line at infinity of $\mathscr{A}$. Also (Kallaher [6]) $G$, and hence $G_{O}$, has the following possible orbit structures on $l_{\infty}$ :

(I) $G$ has one orbit on $l_{\infty}$; i.e., $G$ is transitive on $l_{\infty}$.

(II) $G$ has two orbits on $l_{\infty}$.

(a) One orbit consists of one point and the second orbit consists of the remaining points.

(b) One orbit consists of two points and the second orbit consists of the remaining points.

(c) Both orbits have more than two points.

We will refer to these possible orbit structures in the rest of the paper. Note that all four possibilities actually occur in non-Desarguesian planes.

LEMMA 1. If $\mathscr{A}$ is a finite affine plane of order $n$ with a rank 3 collineation group $G$ which has an orbit structure of type (II) on the line $l_{\infty}$, then for a 
point $O$ of $\mathscr{A}$ :

$$
\begin{aligned}
& \left|G_{O}\right|=k(n-1)\left|G_{O . R}\right|, \quad R \in \Sigma_{1}, \\
& \left|G_{O}\right|=l(n-1)\left|G_{O . Q}\right|, \quad Q \in \Sigma_{2} \text {, }
\end{aligned}
$$

where $k+l=n+1$ and $\Sigma_{1}, \Sigma_{2}$ are the nontrivial orbits of $G_{O}$ in $\mathscr{A}$.

Proof. $G$ having two orbits on $l_{\infty}$ implies $G_{O}$ has the same two orbits on $l_{\infty}$ and thus $G_{O}$ is not transitive on the lines through $O$. This implies that if $l$ is a line of $\mathscr{A}$ through $O$, then the points of $\mathscr{A}$ not equal to $O$ and lying on $l$ must all belong to exactly one of the orbits $\Sigma_{1}, \Sigma_{2}$ of $G_{O}$. Let $\Sigma_{i}$ be the set of all lines through $O$ whose points belong to $\Sigma_{i}, i=1,2$, and let $\Gamma=\left\{l \cap l_{\infty} \mid l \in \bar{\Sigma}_{1}\right\}, \Delta=\left\{l \cap l_{\infty} \mid l \in \bar{\Sigma}_{2}\right\},|\Gamma|=k,|\Delta|=l$. Then $k+l=$ $n+1,\left|\Sigma_{1}\right|=k(n-1),\left|\Sigma_{2}\right|=l(n-1)$. Equations (2.1) follow.

If the rank 3 collineation group $G$ induces a regular permutation group $\bar{G}$ on one of its orbits, say $\Gamma$, then for a point $P \in \Gamma$ we have $G_{P}=G_{\Gamma}$. Since the permutation group $\bar{G}$ induced on $\Gamma$ by $G$ is isomorphic to $G / G_{\Gamma}$, we have $G_{P}$, is a normal subgroup of $G$. This gives:

LEMMA 2. Let $\mathscr{A}$ be a finite affine plane of order $n$ with a rank 3 collineation group $G$. If $G$ induces a regular permutation group on one of its orbits of $l_{\infty}$ and $P$ is a point of that orbit, then $G_{1}$, is normal in $G$.

A special group which we will have use of is the group $T\left(p^{r}\right)$. This is defined as follows:

Definition. Let $p$ be a prime, $r$ an integer $\geqq 1$, and let $V$ be the additive group of $\operatorname{GF}\left(p^{r}\right)$ considered as a vector space over $\operatorname{GF}(p) . T\left(p^{r}\right)$ is the set of all linear transformations of the form $x \rightarrow x^{\sigma} a$, where $a \in \mathrm{GF}\left(p^{r}\right), a \neq 0$, and $\sigma$ is a field automorphism of $\mathrm{GF}\left(p^{r}\right)$.

Note that $T\left(p^{r}\right)=A M$, where $A$ is the subgroup of all mappings of the form $x \rightarrow x^{\sigma}, \sigma$ is a field automorphism of $\mathrm{GF}\left(p^{r}\right)$, and $M$ is the subgroup of all mappings of the form $x \rightarrow x a, a \in \mathrm{GF}\left(p^{r}\right)$ and $a \neq 0$. We will need the information about $T\left(p^{r}\right)$ contained in the next lemma. By a p-primitive divisor of $p^{r}-1, p$ a prime, we mean a divisor of $p^{r}-1$ which is relatively prime to $p^{s}-1$ for every positive integer $s$ less than $r$.

LEMMA 3. Let $G$ be a subgroup of $T\left(p^{r}\right)$ and let $M$ be the subgroup of $T\left(p^{r}\right)$ consisting of mappings of the form $x \rightarrow x a, 0 \neq a \in \mathrm{GF}\left(p^{r}\right)$. If $u$ is $a$ prime p-primitive divisor of $p^{r}-1$ and $H$ is a u-subgroup of $G$, then $H \subset G \cap M$ is a characteristic cyclic subgroup of $G$.

Proof. If $\sigma$ is an automorphism of $\operatorname{GF}\left(p^{r}\right)$, the order of the mapping $x \rightarrow x^{\sigma} a$ is a multiple of the order of $\sigma$. If $\sigma \neq 1$, then $\sigma$ has the form $x \rightarrow x^{p^{s}}$, $s<r$, and the order of $\sigma$ divides $r$. If the order of the original mapping is some power of the prime $u$, then $u \mid r$. Thus $r=u t$. On the other hand, 
since $u$ is a prime, $p^{u-1}-1 \equiv 0(\bmod u)$. Since $u-1<r$ we have a contradiction to the fact that $u$ is a $p$-primitive divisor of $p^{r}-1$.

Hence every element of $G$ whose order is a power of $u$ belongs to $G \cap M$. $G \cap M$ cyclic implies $G \cap M$ contains a unique (cyclic) subgroup of order $k$ for every $k$ dividing $|G \cap M|$. This gives the lemma.

3. The basic result. In this section we prove the following theorem:

THEOREM 2. Let $\mathscr{A}$ be a finite affine plane of order $n \neq 2^{6}, 3^{4}$ with a rank 3 collineation group $G$. If $G$ induces a regular permutation group on one of its orbits on $l_{\infty}$ having length greater than 2 and for a point $P$ of this orbit $G_{1}$, is solvable, then $\mathscr{A}$ is a Desarguesian plane.

ProOf. By Theorem $1, n=p^{r}$ for some prime $p$ and some integer $r \geqq 1$, $\mathscr{A}$ is a translation plane, and $G=T G_{O}$, where $O$ is an affine point of $\mathscr{A}$ and $T$ is the group of translations of $\mathscr{A}$. Furthermore $G$ and $G_{O}$ have the same action on the line $l_{\infty}$. Let $\Gamma$ be the orbit of $G$ (and hence of $G_{O}$ ) on $l_{\infty}$ upon which $G$ (and $G_{O}$ ) acts regularly.

If $\Gamma$ is all of $l_{\infty}$ then the theorem follows from the Corollary in Higman [4]. Hence we may assume $G$ has two orbits on $l_{\infty}$; let $\Delta$ be the second orbit on $l_{\infty}$. If $k=|\Gamma|$ and $l=|\Delta|$, then $k+l=p^{r}+1$.

If $P$ is a point of $l_{\infty}$ then $G_{O . I}$, is transitive on the points of $O P$ other than $O$ and $P$ (proof of Lemma 1). By a proof analogous to that for Lemma 1 of [7], $G_{O, P}$ induces a group $\bar{G}$ of linear transformations on $(Q,+)$ (where $Q$ is a quasi-field coordinatizing $\mathscr{A}$ with $P$ as the point $(\infty)$ and $O$ as the point $(0,0)$ ) considered as a vector space over $\operatorname{GF}(p)$. Note that $G_{O . P}$, $P \in \Gamma$, contains no perspectivities with axis $O P$ since $G_{O}$ acts regularly on $\Gamma$ and $|\Gamma|>2$. Thus $G_{O .1^{P}}$ is isomorphic with $\bar{G}$, a subgroup of $\mathrm{GL}(r, p)$.

We break the proof up into three cases:

Case I. $p^{r}=3^{2}, 5^{2}, 7^{2}, 11^{2}$, or $23^{2}$. If $p^{r}=3^{2}$, then $\mathscr{A}$ is Desarguesian since the only non-Desarguesian translation plane of order 9 is the nearfield plane of that order (Dembowski [3, p. 196]) and that has no collineation group with the prescribed properties. The arguments for the cases $p^{r}=$ $5^{2}, 7^{2}, 11^{2}, 23^{2}$ are similar, so we will give the argument for $p^{r}=5^{2}$ and leave the others for the reader.

Since $G_{O . P}, P \in \Gamma$, is isomorphic to a group of linear transformations on a vector space of dimension 2 over GF(5) we can think of $G_{O . p^{1}}$, as a subgroup of $\operatorname{GL}(2,5)$ which has order $480=5 \cdot 3 \cdot 2^{5}$. Since $\left|G_{O . P}\right|=$ $24 \cdot\left|G_{O . R}\right|, R$ an affine point on $O P,\left|G_{O . R}\right| \mid 20$. If $Q$ is an affine point such that $O Q \cap l_{\infty} \in \Delta$, then the group $G_{O . Q}$ may contain homologies with axis $O Q$. Such homologies must also have their center in $\Delta$. Hence, if $H_{Q}$ is the group of homologies with axis $O Q$, then $\left|G_{O, Q}\right|\left|H_{Q}\right|^{-1} \mid 20$. Note also that if $\sigma \in H_{Q}, \sigma$ fixes no points of $\Gamma$. 
From (2.1) we have

$$
\left|G_{O}\right|=24 \cdot k \cdot\left|G_{O, R}\right|=24 \cdot l \cdot\left|G_{O, Q}\right|,
$$

where $R$ and $Q$ are affine points such that $O R \cap l_{\infty} \in \Gamma, O Q \cap l_{\infty} \in \Delta$, and $k+l=26$. If $l \mid k$ then $k=l=13$. Let $P$ be a point of $\Gamma, Q$ a point of $\Delta$, and let $\Phi$ be the orbit of $Q$ under $G_{O, P}$. Since $G_{O, P}$ is normal in $G_{O}$ (Lemma 2) all orbits of $G_{O, P}$ in $\Delta$ have the same length and thus $|\Phi| \mid 13$. On the other hand $|\Phi||| G_{O . P} \mid$. Since $\left|G_{O, P}\right| \mid 480$, this implies $|\Phi|=1$. Thus $G_{O . P}$ fixes every point of $\Delta$. Since it also fixes every point of $\Gamma, G_{O . P}$ consists of $\left(O, l_{\infty}\right)$-homologies. Thus $\mathscr{A}$ has at least $24=5^{2}-1\left(O, l_{\infty}\right)$-homologies and this implies $\mathscr{A}$ is Desarguesian (Dembowski [3, p. 132]).

Assume $l \nmid k$ and let $d=$ g.c.d. $(l, k), l=u d, k=v d$. Then $u|| G_{O . R} \mid$, $v|| G_{O . Q} \mid$. Thus $u \mid 20$ and $v|20| H_{Q} \mid$. If $u=1$, then $k=25$ and this implies $G_{O, P}$ contains $24\left(O, l_{\infty}\right)$-homologies which implies $\mathscr{A}$ is Desarguesian. $u \neq 20$ since this would imply $l=40$. We consider the other possible values for $u$ and show that in each case a contradiction arises.

If $u=2$, then $d=2, l=4, k=22, v=11$. Since $11 \nmid 20$ we have 11||$H_{Q} \mid$. But then $\mathscr{A}$ would possess a homology of order 11 fixing exactly two points of $\Delta$ and interchanging the other two points-a contradiction. If $u=4$, then $v=9$ and thus 9||$H_{Q} \mid$ which leads to a contradiction since $|\Delta|=l=8$.

If $u=5$, then either $l=5, d=1$ or $l=10, d=2$. For the first possibility $v=21$ which implies 21||$H_{Q} \mid$ and $\mathscr{A}$ would have a homology of order 7 with axis $O Q$ fixing two points of $\Delta$ and permuting the other three-a contradiction. For the second possibility $l=10 \mathrm{implies} 5|| G_{O . R} \mid$ and hence 120||$G_{O . P} \mid, P \in \Gamma . G_{O . P}$ is isomorphic to a subgroup $\bar{G}$ of $\mathrm{GL}(2,5)$. If $\eta: \operatorname{GL}(2,5) \rightarrow \operatorname{PGL}(2,5)$ is the natural homomorphism, then the group $G \eta \cap P S L(2,5)$ has order divisible by 15 and is solvable (since $G_{O, P}$, is). But no solvable subgroup of $\operatorname{PSL}(2,5)$ is divisible by 15 (Huppert [5, p. 213]).

If $u=10$, then $l=20$ and 240||$G_{O . l^{\prime}} \mid, P \in \Gamma$. Arguing as in the case $u=5, l=10$ we get the same contradiction.

Case II. $r=2$ and $p=2^{x}-1$ for $x \geqq 4$. By Passman [11, Theorem 19.9] $\bar{G}$, and hence $G_{O . P}$ for $P \in l_{\infty}$, is isomorphic to a subgroup of $T\left(p^{2}\right)$. $\left|G_{O . P}\right|=\left(p^{2}-1\right)\left|G_{O . R}\right|, R$ an affine point of $O P$, and $\left|T\left(p^{2}\right)\right|=2\left(p^{2}-1\right)$. Hence $\left|G_{O . R}\right| \mid 2$.

If $k=|\Gamma|, l=|\Delta|, d=$ g.c.d. $(k, l), l=d l_{1}, k=d k_{1}$, then from (2.1) we have $l_{1}|| G_{O, R}\left|, k_{1}\right|\left|G_{O . Q}\right|$. If $l_{1}=2$, then $l=2 d$ and $\left|G_{O}\right|=2\left(p^{2}-1\right) k=2 d\left(p^{2}-1\right)$. Hence $k_{1}=1$. Thus $3 d=p^{2}+1, p^{2} \equiv-1(\bmod 3)$. But $p^{2} \equiv 1(\bmod 3)$ for every prime $p$. Hence $l_{1}=1$. If $k_{1}=2$ we obtain the same contradiction. Hence $k_{1}=1$ and $l=k=\frac{1}{2}\left(p^{2}+1\right)$.

$G_{O . P}, P \in \Gamma$, acts as a permutation group on $\Delta$ (not necessarily faithfully). Let $Q \in \Delta$ and let $\Phi$ be the orbit of $Q$ under $G_{O . P}$. Since $G_{O . P^{P}}$ is 
normal in $G$ (Lemma 2), all orbits of $G_{O . P}$ in $\Delta$ have the same length and thus $|\Phi| \mid l=\frac{1}{2}\left(p^{2}+1\right)$. On the other hand, $|\Phi||| G_{O, P} \mid$ and g.c.d. $\left(2\left(p^{2}-1\right), \frac{1}{2}\left(p^{2}+1\right)\right)=1$. Hence $|\Phi|=1$ and $G_{O . P}$ fixes every point of $\Delta$. This implies $G_{O, P}$ consists of $\left(O, l_{\infty}\right)$-homologies (since $G_{O, P}$ fixes every point of $\Gamma$ ) and therefore $\mathscr{A}$ is Desarguesian since it possesses $\left(p^{2}-1\right)$ $\left(O, l_{\infty}\right)$-homologies (Dembowski [3, p. 132]).

Case III. We now complete the proof of the theorem. Because of Cases I and II we may assume $p^{r} \neq 3^{2}, 5^{2}, 7^{2}, 11^{2}, 23^{2}$ and if $r=2, p \neq 2^{x}-1$. Again by Passman [11, Theorem 19.9] for a point $P \in l_{\infty}, G_{O . P}$ is isomorphic to a subgroup of $T\left(p^{r}\right)$. Let $u$ be a prime $p$-primitive divisor of $p^{r}-1$ ( $u$ exists since $p^{r} \neq 2^{6}$, and if $r=2, p \neq 2^{x}-1$; Birkhoff and Vandiver [1] or Zsigmondy [14]). $G_{O . P}$ has a unique subgroup $H$ of order $u$ which is cyclic and characteristic in $G_{O . P}$ (Lemma 3). $G_{O . P}$ normal in $G_{O}$ (Lemma 2) implies $H$ is normal in $G_{O}$ (Schenkman [13, p. 84]).

Let $\sigma$ be an element of $H, \sigma \neq 1$. The fixed points of $\sigma$ and $H$ on $l_{\infty}$ are the same. Assume first that $\sigma$, and hence $H$, fix no points of $\Delta$. Then $|\sigma|=u|l=| \Delta \mid$. From (2.1) we have $u|| G_{O . R} \mid$, since $u \nmid p^{r}+1$ implies $u \nmid k=$ $\left(p^{r}+1\right)-l$. Thus, if $u^{a}$ is the highest power of $u$ dividing $p^{r}-1$, $u^{a+1}|| G_{O, P} \mid, P \in \Gamma$. But $G_{O, P}$ is isomorphic to a subgroup of $T\left(p^{r}\right)$ and $\left|T\left(p^{r}\right)\right|=r\left(p^{r}-1\right)$. Since $u \nmid r$ (see proof of Lemma 3$), u^{a+1} \nmid r\left(p^{r}-1\right)$ and we have a contradiction. Thus $\sigma$, and hence $H$, must fix a point of $\Delta$.

Since $H$ is normal in $G_{O}, H$ must fix every point of $\Delta$. Therefore $H$ consists of $\left(O, l_{\infty}\right)$-homologies since $H$ fixes every point of $\Gamma$ ( $H$ is a subgroup of $\left.G_{O, P}\right)$. But then, $|H|=u|| K \mid$, where $K$ is the kernel of $\mathscr{A}$. Since $|K|=p^{s}-1$ for some $s<r$ (Dembowski $[3$, p. 132]), and $u$ is a $p$-primitive divisor of $p^{r}-1, s=r$ and $|K|=p^{r}-1$. Hence $\mathscr{A}$ is Desarguesian.

4. Proof of the Main Theorem. Our Main Theorem will be a corollary of the following theorem:

THEOREM 3. Let $\mathscr{A}$ be a finite affine plane of order $n$ with a rank 3 collineation group $G$. Assume $n \neq 5^{2}, 11^{2}, 23^{2}, 2^{6}, 3^{4}$, and $n \neq\left(2^{x}-1\right)^{2}$ for some $x \geqq 3$. If $G$ acts regularly on one of its orbits on $l_{\infty}$ and for a point $P$ of this orbit $G_{P}$ is solvable, then $\mathscr{A}$ is either (i) a Desarguesian plane, (ii) $a$ semifield plane, or (iii) a generalized André plane.

Proof. By Theorem $1, \mathscr{A}$ is a translation plane, $n=p^{r}$ for some prime $p$ and some integer $r \geqq 1$, and $G=T G_{O}, T$ the group of translations of $G$ and $O$ an affine point of $\mathscr{A}$. Furthermore $G$ and $G_{O}$ induce the same permutation group on $l_{\infty}$. Let $\Gamma$ be the hypothesized orbit of $G_{O}$ on $l_{\infty}$. If $|\Gamma|>2$ then, by Theorem $2, \mathscr{A}$ is Desarguesian. Thus we may assume $G_{O}$ has either the orbit structure (IIa) or the orbit structure (IIb) on $l_{\infty}$ (see $\S 2)$. We consider each case separately:

Case I. $G_{O}$ has orbit structure (IIa) on $l_{\sigma}$. We shall show that in this 
case $\mathscr{A}$ is either a semifield plane or is Desarguesian. Either $|\Gamma|=1$ or $|\Gamma|=p^{r}$. If it is the latter then, by Theorem 2, $\mathscr{A}$ is Desarguesian. Assume $|\Gamma|=1$. Then, by Corollary 5.1 of Kallaher [7], $\mathscr{A}$ is a semifield plane.

Case II. $G_{O}$ has orbit structure (IIb) on $l_{\infty}$. We shall show that $\mathscr{A}$ is either Desarguesian or a generalized André plane. Either $|\Gamma|=p^{r}-1$ or $|\Gamma|=2$. If $\Gamma$ has length $p^{r}-1$ then, by Theorem $2, \mathscr{A}$ is Desarguesian.

Assume $|\Gamma|=2$. By our assumption on $n=p^{r}, p^{r}-1$ has a prime $p$ primitive divisor. Furthermore $|\Gamma|=2$ implies that, for $P \in \Gamma, G_{P}$ has index 2 in $G$. Hence $G_{P}$ solvable implies $G$ is solvable. By Theorem 4.2 of Kallaher and Ostrom [8], $\mathscr{A}$ is a generalized André plane.

Corollary 3.1. Let $\mathscr{A}$ be a finite plane of order $n$ with a rank 3 collineation group $G$. If $n$ is not a square and $G$ acts regularly on one of its orbits on the line at infinity, then $\mathscr{A}$ is either (i) a Desarguesian plane, (ii) a semifield plane, or (iii) a generalized André plane.

Proof. Let $\Gamma$ be the orbit on $l_{\infty}$ upon which $G$ acts regularly. If $|\Gamma|=1$ the theorem follows from Kallaher [7]. We therefore assume $|\Gamma|>1$. We wish to apply Theorem 3. Because $n$ is not a square, the hypothesis in Theorem 3 concerning $n$ is satisfied. Let $P$ be a point of $\Gamma$ and $Q$ another point of $\Gamma$. Then $G_{P}=G_{P, Q}$ since $G$ acting regularly on $\Gamma$ implies $G_{P}$, fixes all points of $\Gamma$. By Burmeister and Hughes [2, corollary, p. 182] $G_{P, Q}$ is solvable. Thus the corollary follows from Theorem 3 .

\section{REFERENCES}

1. G. D. Birkhoff and H. S. Vandiver, On the integral divisor of $a^{n}-b^{n}$, Ann. of Math. 5 (1904), 173-180.

2. M. V. D. Burmeister and D. R. Hughes, On the solvability of autotopism groups, Arch. Math. 16 (1965), 178-183. MR 31 \#2330.

3. P. Dembowski, Finite geometries, Ergebnisse der Mathematik und ihrer Gienzgebiete, Band 44, Springer-Verlag, Berlin and New York, 1968. MR 38 \#1597.

4. D. G. Higman, On finite affine planes of rank 3, Math. Z. 104 (1968), 147-149. MR 36 \#7018.

5. B. Huppert, Endliche Gruppen. I, Die Grundlehren der math. Wissenschaften, Band 134, Springer-Verlag, Berlin and New York, 1967. MR 37 \#302.

6. M. J. Kallaher, On finite affine planes of rank 3, J. Algebra 13 (1969), 544-553. MR 40 \#832.

7. - A class of rank three affine planes, Math Z. 119 (1971), 75-82.

8. M. J. Kallaher and T. G. Ostrom, Fixed point free linear groups, rank three planes, and Bol quasifields, J. Algebra 18 (1971), 159-178.

9. R. A. Liebler, Finite affine planes of rank three are translation planes, Math. Z. 116 (1970), 89-93. MR 42 \#948.

10. H. Lüneburg, Über projektive Ebenen, in denen jede Fahne von einer nichttrivialen Elation invariant gelassen wird, Abh. Math. Sem. Univ. Hamburg 29 (1965), 37-76. MR 32 \#4594. 
11. D. S. Passman, Permutation groups, Benjamin, New York, 1968.

12. O. Prohaska, Endliche ableitbare affine Ebenen, Geometriae Dedicata (to appear).

13. E. Schenkman, Group theory, Van Nostrand, Princeton, N.J., 1965. MR 33 \#5702.

14. K. Zsigmondy, Zur Theorie der Potenzreste, Monatsh. Math. Phys. (1892), 265-284.

Department of Mathematics, Washington State University, Pullman, WashINGTON 99163 